OPEN ACCESS

Edited by:

Anette S. B. Wolff,

University of Bergen, Norway

Reviewed by:

Hsiuying Wang,

National Yang Ming Chiao Tung

University, Taiwan

Stefan Blum,

The University of Queensland,

Australia

*Correspondence:

Qishui Ou

ouqishui@fjmu.edu.cn

${ }^{t}$ These authors have contributed equally to this work and share

first authorship

Specialty section: This article was submitted to

Autoimmune and Autoinflammatory Disorders,

a section of the journal

Frontiers in Immunology

Received: 20 December 2021 Accepted: 25 January 2022

Published: 22 February 2022

Citation:

Liu X, Fan K, Lin Q, Tang M, Wang Q, Huang E, Zhang W, Chen T and Ou Q (2022) Serum-Derived Exosomal miR140-5p as a Promising Biomarker for Differential Diagnosis of Anti-NMDAR Encephalitis With Viral Encephalitis.

Front. Immunol. 13:840003. doi: 10.3389/fimmu.2022.840003

\section{Serum-Derived Exosomal miR-140- $5 p$ as a Promising Biomarker for Differential Diagnosis of Anti-NMDAR Encephalitis With Viral Encephalitis}

\author{
Xiaofeng Liu ${ }^{1,2,3 \dagger}$, Kengna Fan ${ }^{1 \dagger}$, Qingwen Lin ${ }^{1}$, Minjie Tang ${ }^{1}$, Qi Wang ${ }^{1}$, Er Huang ${ }^{1}$, \\ Weiqing Zhang ${ }^{1}$, Tianbin Chen ${ }^{1,2,3}$ and Qishui Ou ${ }^{1,2,3 *}$
}

\begin{abstract}
1 Department of Laboratory Medicine, the First Affiliated Hospital of Fujian Medical University, Fuzhou, China, 2 Fujian Key Laboratory of Laboratory Medicine, The First Affiliated Hospital, Fujian Medical University, Fuzhou, China, ${ }^{3}$ Gene Diagnosis Research Center, the First Affiliated Hospital of Fujian Medical University, Fuzhou, China
\end{abstract}

Background: Anti- $N$-methyl-D-aspartate receptor (anti-NMDAR) encephalitis is the most common type of autoimmune encephalitis. Early recognition and treatment, especially distinguishing from viral encephalitis (VE) in the early stages, are crucial for the outcomes of patients with anti-NMDAR encephalitis. Compared with plasma microRNAs (miRNAs), exosomal miRNAs are more abundant and not easy to degrade. Moreover, exosomes can pass through the blood-brain barrier. This study aimed to explore the clinical value of serum exosomal miRNAs in the differential diagnosis of anti-NMDAR encephalitis with VE.

Method: Serum samples from a total of 30 patients with anti-NMDAR encephalitis, 30 patients with VE, and 30 cases of control patients hospitalized in the same period were collected. Firstly, the serum exosomes were isolated and identified by transmission electron microscope (TEM), nanoparticle-tracking analyzer (NTA), and Western blot (WB). The expression levels of let-7b and miR-140-5p from serum exosomes were detected by real-time quantitative PCR (qPCR). At the same time, we also detected complement 3 (C3), complement 4 (C4), and high sensitivity CRP (hs-CRP) expression levels in three groups. Finally, we analyzed the difference and diagnostic value of the test results.

Results: Isolated particles showed identical characteristics to the exosomes through TEM, NTA, and WB analyses. Compared with the VE group and control group, the expression of miR-140-5p was significantly upregulated in serum exosomes of the NMDAR group. In contrast, the serum C3 in the NMDAR group was significantly lower than the other two groups. ROC curve analysis showed the area under the curve (AUC) of serum exosomal miR-140-5p and serum C3 was 0.748 (76.67\% sensitivity and $73.33 \%$ specificity) and 0.724 (76.67\% sensitivity and 60\% specificity) to distinguish anti-NMDAR encephalitis from VE, respectively. The AUC of serum exosomal miR-140-5p combined with serum C3 was 0.811 , the sensitivity was $70.00 \%$, and the specificity was $86.67 \%$. 
Conclusion: Serum exosomal miR-140-5p combined with serum C3 would be a promising marker in the differential diagnosis of anti-NMDAR encephalitis with VE.

Keywords: anti-NMDAR encephalitis, viral encephalitis, exosome, miRNAs, biomarker

\section{INTRODUCTION}

Autoimmune encephalitis (AE) is an inflammatory disease of the central nervous system in which antibodies mediate neuronal damage and cause structural dysfunction (1). Anti- $N$-methyl-Daspartate receptor encephalitis (anti-NMDAR encephalitis) is the most common subtype of AE (1), which was characterized by a series of complex neuropsychiatric symptoms and expresses antibodies against subunits of NMDAR GluN1 in CSF (2). The clinical manifestations of anti-NMDAR encephalitis are diverse, and the electroencephalogram (EEG) and imaging examinations lack specificity. The diagnosis is mainly reliant on the positive result of CSF or serum antibodies. However, antibody examinations were not popularized and some hospitals need to send them to a third-party institution for testing, which takes about 3-5 days, delaying diagnosis and worsening patients' economic and emotional burden (3). In addition, the clinical symptoms, cerebrospinal fluid (CSF) tests, and brain MRI are similar between the disease of $\mathrm{AE}$ and VE. The detection rate of $\mathrm{VE}$ pathogens is low; therefore, VE is mainly diagnosed on the basis of epidemiology, clinical manifestations, CSF detection, imaging, EEG and of excluding other diseases. In conclusion, it is difficult to diagnose patients with anti-NMDAR encephalitis in the early stage.

Exosomes are small vesicles of about $30-150 \mathrm{~nm}$ in body fluid, mainly formed by invagination of lysosomal microparticles in the cell, rich in RNA and protein participating in intercellular communication (4). MicroRNA (miRNA) is a highly conservative short-chain noncoding RNA that plays a vital role in the central nervous system. It has been studied in various central nervous system diseases such as ischemic stroke, Alzheimer's disease, and tuberculous meningitis (5-7). It has been confirmed that blood miRNAs are mainly derived from exosomes. Compared with plasma miRNAs, exosomal miRNAs are more abundant and stable (8). In addition, exosomes can pass through the blood-brain barrier, so the serum exosomal miRNAs can also reflect the characteristics of miRNAs in the central nervous system (9), which is a promising marker for neurological diseases.

Recent studies have shown that let-7b and miR-140-5p play a certain role in the central nervous system and participate in the processes of apoptosis, tumorigenesis, and inflammatory progression of nerve cells (10-12). However, these above miRNA-derived serum exosomes have not yet been reported in the anti-NMDAR encephalitis or VE. This study aimed to isolate serum exosomes and explore the value of serum exosomal let-7b and miR-140-5p in the anti-NMDAR encephalitis, especially the value of differential diagnosis between anti-NMDAR encephalitis and VE.

\section{MATERIALS AND METHODS}

\section{Study Subjects}

From October 2018 to November 2020, we collected data from 30 cases of anti-NMDAR encephalitis, 30 cases of VE, and 30 cases of noninflammatory neurologic disorders as control patients with matching age and sex in the first affiliated Hospital of Fujian Medical University (including mental disorder, cerebrovascular disease, neurodegenerative diseases, respiratory tract infection, syphilis not involving the central nervous system, ataxia, dyskinesia, peripheral neuropathy, and so on).

We collected $5 \mathrm{ml}$ serum stored at $-80^{\circ} \mathrm{C}$ for further analysis and clinical data of the above three groups of patients. This study has been approved by the Ethics Committee of the First Affiliated Hospital of Fujian Medical University (No, MRCTA, ECFAH of FMU, 2020[324]). Each participant signed written informed consent before recruitment.

\section{Exosome Isolation and Identification}

The Serum Exosome Isolation Kit (\#UR52136, Umibio Co., Ltd., Shanghai, China) was used to isolate exosomes according to the manufacturer's instructions. Finally, 200- $\mu$ l suspension solution was obtained and stored at $-80^{\circ} \mathrm{C}$ for further analysis. Transmission electron microscopy (TEM), nanoparticle-tracking analysis (NTA), and Western blot (WB) analysis including CD63 and TSG101 were used to confirm the characters of exosomes.

\section{Detection of Serum Exosomal miRNA}

Firstly, according to the experimental procedure by the miRNA Purification Kit (CW0627, CWBIO, Taizhou, China), $200 \mu \mathrm{l}$ exosomes eventually obtained $30 \mu \mathrm{l}$ total miRNA. Secondly, miRNA 1st Strand cDNA Synthesis Kit (MR101-01, Vazyme, Nanjing, China) was used to reverse transcripted cDNAs with a stem-loop RT primer. All primers were synthesized by Sangon Biotech Co., Ltd. (Shanghai, China), and primer sequence information is shown in Table 1. Finally, exosomal miRNAs (let-7b and miR-140-5p) were measured via real-time PCR using miRNA Universal SYBR qPCR Master Mix (MQ101-01, Vazyme, Nanjing, China). U6 was used as the internal reference, and cel-miR-39 was used as the external reference. PCR reaction was performed in triplicate and analyzed using a QuantStudio Dx qPCR system (Applied Biosystems, Foster City, CA, USA).

\section{Detection of Serum Complement and hs-CRP}

The levels of serum complement 3 (C3) and complement 4 (C4) were measured on IMMAGE 800 Immunochemistry System 
TABLE 1 | Gene primers.

\begin{tabular}{|c|c|c|}
\hline Name & RT primers $\left(5^{\prime}-3^{\prime}\right)$ & Forward primers $\left(5^{\prime}-3^{\prime}\right)$ \\
\hline U6 & AACGCTTCACGAATTTGCGT & CTCGCTTCGGCAGCACA \\
\hline let-7b & GTCGTATCCAGTGCAGGGTCCGAGGTATTCGCACTGGATACGACAACCAC & GCGCGTGAGGTAGTAGGTTGT \\
\hline miR-140-5p & GTCGTATCCAGTGCAGGGTCCGAGGTATTCGCACTGGATACGACCTACCA & CGCGCAGTGGTITACCCTA \\
\hline Universal reverse primers & AGTGCAGGGTCCGAGGTATT (5'-3') & \\
\hline
\end{tabular}

(Beckman Coulter, Brea, CA, USA). High-sensitivity CRP (hsCRP) level was measured on ADVIA 2400 Chemistry System (Siemens Healthineers, Erlangen, Germany).

\section{Statistics}

Normally distributed data was expressed as the mean \pm standard deviation (mean $\pm \mathrm{SD}$ ), and Student's t-test was used to compare the two groups. One-way analysis of variance was used for the comparison among the multiple groups. In nonnormally distributed data, the median and interquartile range (m, Q) was used to show the characteristic of the data, and the MannWhitney $U$ test was used to compare the outcomes. The KruskalWallis $H$ test was used for the comparison among the multiple groups. The relative expression values were normalized to U6 and miR-39 and calculated by the $2^{-\Delta \Delta C t}$ method. The receiver operating characteristic (ROC) curve was performed by the MedCalc system. $p<0.05$ was statistically significant.

\section{RESULTS}

\section{Clinical Characteristics of Subjects}

In our study, we enrolled 30 patients with NMDAR, 30 patients with VE, and 30 age- and sex-matched control group (CG), respectively. As shown in Table 2, age and sex were not significantly different among the three groups. Seizure (63.3\%) was the most common clinical presentation in the NMDAR group, while fever was the most common clinical presentation in the VE group (66.7\%). White blood cell count in CSF of patients was notably increased in the NMDAR group and VE group compared with the CG group. All patients in the NMDAR group had positive result in the test of serum or CSF antibody. Only two anti-NMDAR patients had teratoma comorbidity, while one VE patient had hypophysoma. Some patients in each group had been associated with infectious diseases.

\section{Characterization of the Isolated Serum Exosomes}

Serum-derived vesicles showed a circular, round, and cup morphology with a diameter of about $30-150 \mathrm{~nm}$ by TEM. The vesicles were coated with a phospholipid bimolecular layer and contained a low electron density substance (Figure 1A). Furthermore, the NTA measurements revealed that our serum particles' average size and main peak were about $100 \mathrm{~nm}$, and the concentration was $5.9 \times 10^{9} / \mathrm{mL}$ (Figure 1B). Moreover, the exosome markers, including CD63 and TSG101, were positively expressed in the isolated particles, with low expression of GAPDH. The expression of the above protein in the mononuclear cell was opposite, where the high expression of GAPDH and no expression of CD63 and TSG101 are shown (Figure 1C). The characteristics described above are consistent with exosomes (4), indicating that we have successfully obtained exosomes from serum.

\section{The Expression Levels of let-7b and miR-140-5p in Serum Exosomes}

Expression levels of let-7b in the NMDAR, VE, and CG groups were analyzed. As shown in Figure 2, the relative expression level

TABLE 2 | Clinical characteristics of patients in three groups.

\begin{tabular}{|c|c|c|c|c|}
\hline & NMDAR group & VE group & Control group & $p$-value \\
\hline Total number $(n)$ & 30 & 30 & 30 & - \\
\hline Age (years, mean \pm SD) & $24.3 \pm 13.3$ & $29.2 \pm 17.3$ & $30.6 \pm 20.5$ & 0.341 \\
\hline $\operatorname{Sex}[\mathrm{M}(n, \%)]$ & 15 (50.0\%) & 14 (46.7\%) & 13 (43.3\%) & 0.876 \\
\hline \multicolumn{5}{|l|}{ Clinic symptoms $(n, \%)$} \\
\hline Fever & $8(26.7 \%)$ & 20 (66.7\%) & $8(26.7 \%)$ & 0.001 \\
\hline Psychiatric symptom & 15 (50\%) & $6(20 \%)$ & $3(10 \%)$ & 0.001 \\
\hline Abnormal movements & 15 (50.0\%) & $5(16.7 \%)$ & $4(13.3 \%)$ & 0.002 \\
\hline Disorders of memory & $8(26.7 \%)$ & 1 (3.3\%) & $1(3.3 \%)$ & 0.005 \\
\hline Seizure & 19 (63.3\%) & 7 (23.3\%) & $3(10 \%)$ & $<0.001$ \\
\hline Disorders of sleep ( $n, \%)$ & $5(16.7 \%)$ & 1 (3.3\%) & $1(3.3 \%)$ & 0.099 \\
\hline \multicolumn{5}{|l|}{ CSF routine $[\mathrm{M}(\mathrm{Q} 1, \mathrm{Q} 3)]$} \\
\hline WBC (×106/L) & $13(3.8,30.3)$ & $23(7.8,63.5)$ & $2(1.0,3.3)$ & $<0.001$ \\
\hline GLU (mmol/L) & $2.8(2.6,3.4)$ & $3.08(2.8,3.4)$ & $2.89(2.6,3.4)$ & 0.239 \\
\hline $\mathrm{Cl}(\mathrm{mmol} / \mathrm{L})$ & $119.0(117.0,121.0)$ & $119.5(116.8,123.0)$ & $120.0(119.0,121.0)$ & 0.279 \\
\hline Serum/CSF anti-NMDAR antibody positive ( $n, \%)$ & $30(100 \%)$ & 0 & 0 & - \\
\hline Tumor comorbidity ( $n, \%)$ & $2(6.7 \%)$ & $1(3.3 \%)$ & 0 & 0.355 \\
\hline Infectious disease (n, \%) & $9(30.0 \%)$ & $14(46.7 \%)$ & $11(36.7 \%)$ & 0.407 \\
\hline
\end{tabular}

NMDAR, anti-N-methyl-D-aspartate receptor encephalitis; VE, viral encephalitis; $M$, male. 
A

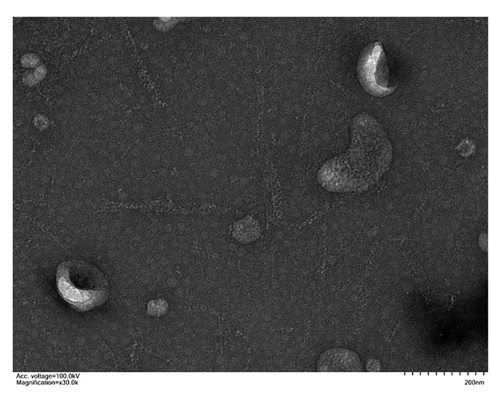

B

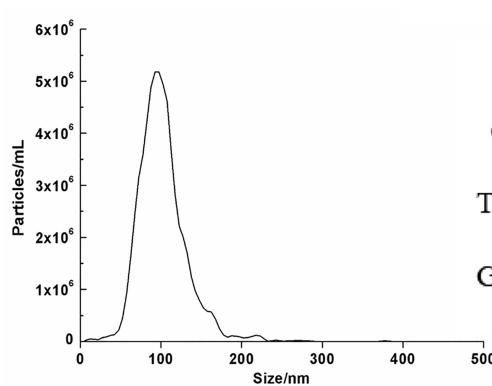

C

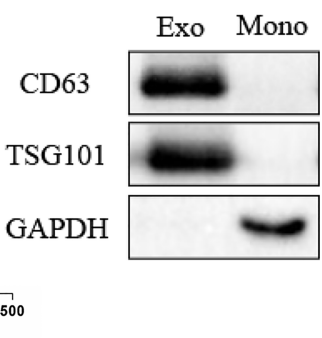

FIGURE 1 | Characterization of the isolated serum exosomes. (A) Morphology of serum exosome by electron transmission microscope ( $\times 30.0 \mathrm{~K})$. (B) Concentration and size distribution of serum exocrine. (C) The expression of the surface-specific protein of exosome by Western blot. (Exo, exosomes; Mono, mononuclear cell; CD63 and TSG101 were exosome markers).

of let-7b in the serum exosomes of the NMDAR group was the highest, but there was no significant difference in the three groups (all $p>0.05$ ). Meanwhile, we analyzed the expression level of miR-140-5p in the three groups. The miR-140-5p expression in the NMDAR group was significantly higher than that in the VE group $(p<0.001)$ and the CG group $(p<0.05)$. In addition, the expression level of miR-140-5p in the CG group was higher than that of the VE group, and the difference was statistically significant (all $p<0.05$ ).

\section{The Expression Levels of Serum C3, C4, and hs-CRP}

The expression of C3, C4, CH50, and CRP in anti-NMDAR encephalitis has been reported as abnormal and correlated with disease severity (13). Therefore, we detected serum C3, C4, and hs-CRP in the three groups. The expression of C3 in the NMDAR group was the lowest and significantly lower than that of the VE group and CG group (NMDAR group:
$0.926 \pm 0.191 \mathrm{~g} / \mathrm{L}$; VE group: $1.119 \pm 0.232 \mathrm{~g} / \mathrm{L}$; CG group: $1.166 \pm 0.193 \mathrm{~g} / \mathrm{L})(p<0.05)$ (Figure 3). The expression of C4 in the NMDAR group was slightly lower than the other two groups, but there was no significant difference (NMDAR group: $0.206 \pm 0.0 .092 \mathrm{~g} / \mathrm{L}$; VE group: $0.234 \pm 0.084 \mathrm{~g} / \mathrm{L}$; CG group: $0.262 \pm 0.115 \mathrm{~g} / \mathrm{L})(p>0.05)$ (Figure 3). There was no significant difference in the expression of hs-CRP in the three groups (NMDAR group: $3.212 \pm 5.601 \mathrm{mg} / \mathrm{L}$; VE group: $4.484 \pm 6.585 \mathrm{mg} / \mathrm{L}$; CG group: $3.643 \pm 6.151 \mathrm{mg} / \mathrm{L}$ ) (all $p>0.05$, Figure 3).

\section{Differential Diagnostic Value of Serum Exosomal miR-140-5p and Serum C3}

We later used the ROC curve to evaluate the differential diagnosis value of miR-140-5p and serum C3 in the NMDAR group against the VE group. The area under the curve (AUC) of serum exosomal miR-140-5p was 0.748 (95\% CI: 0.620-0.851), and the sensitivity was $76.67 \%$ at the specificity of $73.33 \%$.
A

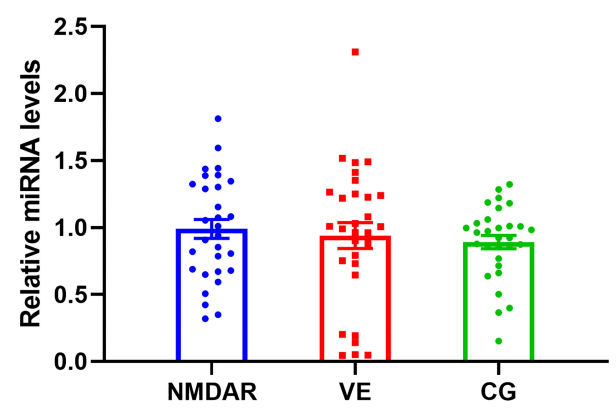

let-7b
B

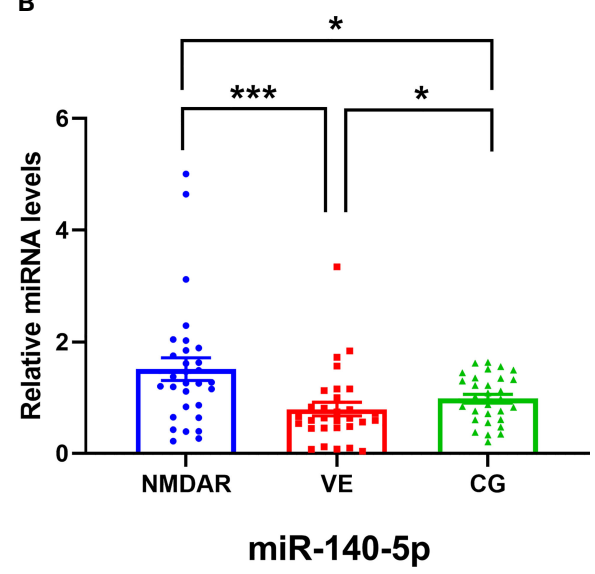

FIGURE 2 | The expression levels of let-7b and miR-140-5p in serum exosomes in three groups. (A) The expression level of let-7b among the NMDAR, VE, and CG groups. (B) The expression level of miR-140-5p among the NMDAR, VE, and CG groups. $\left({ }^{\star \star \star} p<0.001 ;{ }^{*} p<0.05\right.$; NMDAR, anti-N-methyl-D-aspartate receptor encephalitis; VE, viral encephalitis; CG, control group). 
A

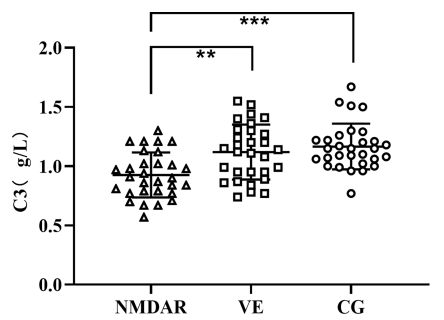

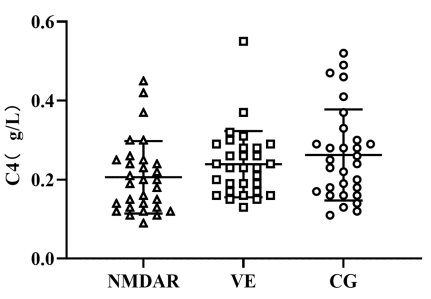

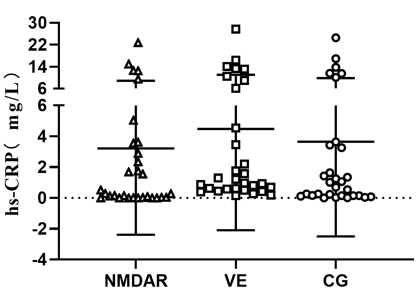

FIGURE 3 | The expression levels of serum C3, C4, and hs-CRP. (A) The expression level of C3 among three groups. (B) The expression level of C4 among three groups. (C) The expression level of hs-CRP among three groups. ( ${ }^{\star \star \star} p<0.001$; ${ }^{\star \star} p<0.01$; NMDAR, anti- $N$-methyl-D-aspartate receptor encephalitis; VE, viral encephalitis; CG, control group; C3, complement 3; C4, complement 4; hs-CRP, high sensitivity CRP).

The AUC of serum C3 alone was 0.724 (95\% CI: 0.594-0.832), and the sensitivity was $76.67 \%$ at the specificity of $60 \%$. The AUC of serum exosomal miR-140-5p combined with serum C3 was 0.811 (95\% CI: $0.661-0.881$ ), and the sensitivity was $70.00 \%$ at the specificity of $86.67 \%$ (Figure 4 ).

\section{DISCUSSION}

Since Dalmau et al. firstly reported anti-NMDAR encephalitis in 2007 (14), the anti-NMDAR encephalitis has become the most common type of $\mathrm{AE}$ in clinical practice. However, the pathogenesis of anti-NMDAR encephalitis remains unclear. There are some hypotheses, such as tumor tissue antigenactivated autoimmune reaction (15), antigen exposure induced after viral infection $(16,17)$, and immune clearance disorder (18). Moreover, the disease progression of anti-NMDAR encephalitis is fast and serious, and early detection can significantly improve the disease and prognosis. However, the clinical manifestations of anti-NMDAR encephalitis are diverse, and laboratory tests, brain MRI, or EEG are nonspecific, similar to that of VE. Therefore, it is still a huge challenge to identify the two diseases before autoantibody or pathogen detection results are confirmed $(16,19)$. Therefore, it is significant to find

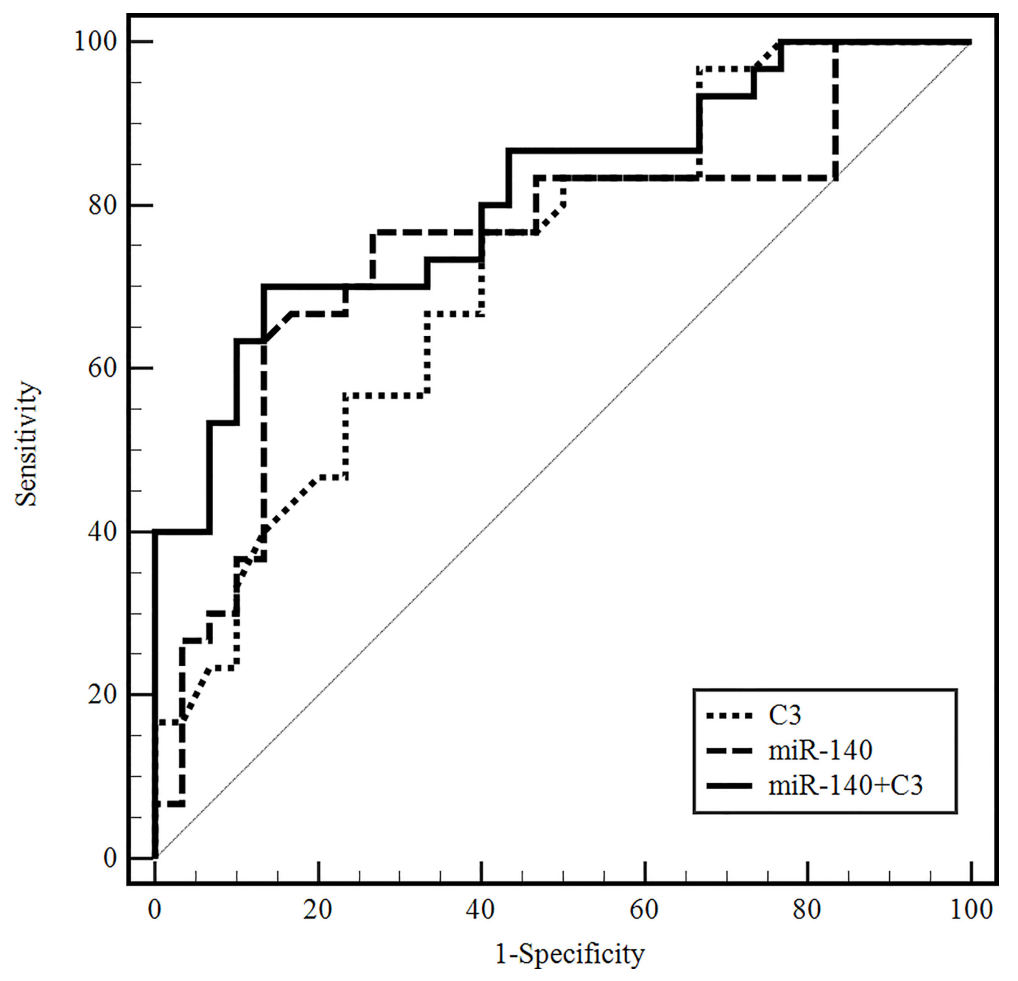

FIGURE 4 | ROC curves to validate the differential diagnostic value of exosomal miR-140-5p, serum C3, and their combinations for anti-NMDAR encephalitis. (C3, complement 3; C4, complement 4; miR-140, microRNA-140-5p). 
biomarkers that can be used for early identification of antiNMDAR encephalitis, especially to distinguish from VE.

To date, few studies have reported the serum exosomal miRNAs in patients with anti-NMDAR encephalitis. Recent studies have shown that exosomal miRNAs are more abundant than plasma miRNAs and are less susceptible to degradation (8). Moreover, exosomes can cross the blood-brain barrier into the peripheral blood, allowing serum exosomal miRNAs to represent central nervous system miRNA properties without invasive examination (9). In this study, we focused on the value of serum exosomal miRNAs as differential diagnostic biomarkers of anti-NMDAR encephalitis. Our results showed that the serum exosomal miR-140-5p expression in the NMDAR group was significantly higher than that in the VE and CG groups. The ROC analysis showed that serum exosomal miR-140-5p combined with serum C3 may serve as a promising biomarker for the differential diagnostic of anti-NMDAR encephalitis.

After separating the serum exosomes, we first used TEM to observe its characteristic, which was about 30-150 nm diameter, with the shape of circular, round and cup, and wrapped with phospholipid double molecular layer and contained low electron density substances internal. Furthermore, NTA showed the serum exosomes' concentration was $5.9 \times 10^{9} / \mathrm{mL}$ and the average diameter was $100 \mathrm{~nm}$. Finally, WB analysis showed a high expression of CD63 and TSG101 in serum exosomal protein. The above results were consistent with the characteristics of the exosomes (4).

Let-7b is a member of the let-7 family and is involved in the development of multiple diseases, such as the let-7b/TLR4 pathway. It can promote macrophage differentiation and chronic inflammation regression and participate in the inflammatory process (20); Zhang et al. (21) showed that the expression of plasma let-7b in the anti-NMDAR encephalitis treatment group was significantly downregulated compared with the control group. However, it is not clear whether the expression of let-7b in the serum exosomes of AE patients was changed. In this study, we found that the expression of let-7b in serum exosomes was not significantly different between the NMDAR, VE, and CG groups. Considering that most of the serum samples in the NMDAR group were in the pretreatment stage and not yet treated with medication, there was no difference in the serum exosomal let- $7 \mathrm{~b}$.

It has been reported that miR-140-5p was associated with many diseases such as cardiocerebral vascular diseases, tumors, autoimmune diseases, etc. Guan et al. (22) found that the expression of miR-140-5p was negatively correlated with multiple sclerosis, and miR-140-5p inhibited CD4+ T-cell differentiation and STAT1 activation, thus slowing the progression of multiple sclerosis. Zhu et al. (23) reported that miR-140-5p was involved in the pathogenesis of autoimmune encephalomyelitis by regulating Th1 differentiation through DNA methylation and mitochondrial pathway. It has been reported that miR-140-5p was involved in the progression of inflammatory and autoimmune diseases, but no scholars have reported the relationship between miR-140-5p and antiNMDAR encephalitis. This study showed that the expression of serum exosomal miR-140-5p was significantly higher in the
NMDAR group than in the VE and CG groups. A recent study had shown that the PI3K-AKT pathway participated in the activation and proliferation of B lymphocytes of systemic lupus erythematosus; PTEN inhibited the occurrence of this pathway (24). Yin et al. (25) showed that miR-140 inhibited PTEN expression. Therefore, we speculated that miR-140-5p might be involved in the activation and proliferation of $\mathrm{B}$ cells via the PI3K-AKT pathway by inhibiting the expression of PTEN in anti-NMDAR encephalitis, and then participated in the production of autoantibodies. We then used the ROC curve to evaluate the diagnostic efficacy of miR-140-5p between the NMDAR and VE groups, which found that the AUC, sensitivity, and specificity were respectively $0.748,76.67 \%$, and $73.33 \%$, whereby the diagnostic efficacy needs to be improved.

Based on the study of miR-140-5p involvement in the immune response described above, C3, C4, and CRP expression in antiNMDAR encephalitis were abnormal and correlated with disease severity (13); we speculated whether miR-140-5p is related to the above complement system. Therefore, we examined the levels of $\mathrm{C} 3$, C4, and hs-CRP among the three groups. The results showed that the expression of C3 in the NMDAR group was the lowest and significant, while the expression of C4 and hs-CRP was not significantly different among the three groups. Subsequently, we found no correlation between miR-140-5p and C3, C4, and hs-CRP.

Next, we further evaluated whether the combination of serum exosomal miR-140-5p and serum C3 could improve the differential diagnosis efficacy against anti-NMDAR encephalitis and VE using the ROC curve analysis. The AUC value of the serum exosomal miR-140-5p combined with serum C3 was 0.811 , with $70.00 \%$ sensitivity and $86.67 \%$ specificity, which was higher than that of serum exosomal miR-140-5p or serum C3, alone.

To our knowledge, this is the first study to explore the possible value of serum exosomal miRNAs as differential diagnostic markers of anti-NMDAR encephalitis. We found a significant association of serum exosomal miRNAs with anti-NMDAR encephalitis. In addition, serving as a biomarker, serum exosomal miRNAs is convenient without invasive examination. At the same time, there are some limitations in this study. The sample size analyzed for each group is relatively small. Additionally, in the present study, we focused on the study of exosomal miRNAs from serum and have not yet been compared with exosomal miRNAs from CSF in patients with anti-NMDAR encephalitis. Future study will be needed to explore the value of CSF exosomal miRNAs in patients with anti-NMDAR encephalitis.

In conclusion, our results suggested that serum exosomal miR-140-5p expression was significantly elevated in patients with anti-NMDAR encephalitis, and serum exosomal miR-140$5 \mathrm{p}$ combined with serum $\mathrm{C} 3$ would be a promising biomarker for the differential diagnosis of anti-NMDAR encephalitis and VE.

\section{DATA AVAILABILITY STATEMENT}

The original contributions presented in the study are included in the article/supplementary material. Further inquiries can be directed to the corresponding author. 


\section{ETHICS STATEMENT}

The studies involving human participants were reviewed and approved by The Ethics Committee of the First Affiliated Hospital of Fujian Medical University. The patients/participants provided their written informed consent to participate in this study.

\section{AUTHOR CONTRIBUTIONS}

XFL and QSO put forward the concept and designed the study. KNF, QWL, and QW performed clinical data and serum sample collection. XFL, KNF, MJT, and QW carried out experiments. EH, WQZ, TBC, and QSO analyzed the data. XFL and KNF wrote the manuscript. XFL and QSO reviewed the manuscript and finalized the paper. All authors read and approved the final manuscript.

\section{REFERENCES}

1. Graus F, Titulaer MJ, Balu R, Benseler S, Bien CG, Cellucci T, et al. A Clinical Approach to Diagnosis of Autoimmune Encephalitis. Lancet Neurol (2016) 15:391-404. doi: 10.1016/s1474-4422(15)00401-9

2. Dalmau J, Rosenfeld MR. Autoimmune Encephalitis Update. Neuro Oncol (2014) 16:771-8. doi: 10.1093/neuonc/nou030

3. Qu X-P, Vidaurre J, Peng X-L, Jiang L, Zhong M., Hu Y. Seizure Characteristics, Outcome, and Risk of Epilepsy in Pediatric Anti-N-MethylD-Aspartate Receptor Encephalitis. Pediatr Neurol (2020) 105:35-40. doi: 10.1016/j.pediatrneurol.2019.11.011

4. Yellon DM, Davidson SM. Exosomes: Nanoparticles Involved in Cardioprotection? Circ Res (2014) 114:325-32. doi: 10.1161/CIRCRESAHA.113.300636

5. Liu CG, Song J, Zhang YQ, Wang PC. MicroRNA-193b Is a Regulator of Amyloid Precursor Protein in the Blood and Cerebrospinal Fluid Derived Exosomal microRNA-193b Is a Biomarker of Alzheimer's Disease. Mol Med Rep (2014) 10:2395-400. doi: 10.3892/mmr.2014.2484

6. Xin H, Yang Y, Liu J, Li X, Li M, Feng B, et al. Association Between Tuberculosis and Circulating microRNA Hsa-Let-7b and hsa-miR-30b: A Pilot Study in a Chinese Population. Tuberculosis (Edinb) (2016) 99:63-9. doi: 10.1016/j.tube.2016.04.005

7. Chen Z, Wang K, Huang J, Zheng G, Lv Y, Luo N, et al. Upregulated Serum MiR-146b Serves as a Biomarker for Acute Ischemic Stroke. Cell Physiol Biochem (2018) 45:397-405. doi: 10.1159/000486916

8. Cheng L, Quek CY, Sun X, Bellingham SA, Hill AF. The Detection of microRNA Associated With Alzheimer's Disease in Biological Fluids Using Next-Generation Sequencing Technologies. Front Genet (2013) 4:150. doi: 10.3389/fgene.2013.00150

9. Lee Y, El Andaloussi S, Wood MJ. Exosomes and Microvesicles: Extracellular Vesicles for Genetic Information Transfer and Gene Therapy. Hum Mol Genet (2012) 21:R125-34. doi: 10.1093/hmg/dds317

10. Song W, Wang T, Shi B, Wu Z, Wang W, Yang Y. Neuroprotective Effects of microRNA-140-5p on Ischemic Stroke in Mice via Regulation of the TLR4/NFkappaB Axis. Brain Res Bull (2021) 168:8-16. doi: 10.1016/j.brainresbull.2020.10.020

11. Wang H. Anti-NMDA Receptor Encephalitis: Efficacy of Treatment for Male Patients and miRNA Biomarker. Curr Med Chem (2020) 27:4138-51. doi: 10.2174/0929867325666180221142623

12. Sorensen SS, Nygaard AB, Nielsen MY, Jensen K, Christensen T. miRNA Expression Profiles in Cerebrospinal Fluid and Blood of Patients With Acute Ischemic Stroke. Transl Stroke Res (2014) 5:711-8. doi: 10.1007/s12975-014-0364-8

13. Shu Y, Chen C, Chen Y, Xu Y, Chang Y, Li R, et al. Serum Complement Levels in Anti-N-Methyl-D-Aspartate Receptor Encephalitis. Eur J Neurol (2018) 25:178-84. doi: 10.1111/ene.13495

14. Dalmau J, Tüzün E, Wu H, Masjuan J, Rossi J, Voloschin A, et al. Paraneoplastic Anti-N-Methyl-D-Aspartate Receptor Encephalitis Associated With Ovarian Teratoma. Ann Neurol (2007) 61:25-36. doi: 10.1002/ana.21050

15. Kreye J, Wenke NK, Chayka M, Leubner J, Murugan R, Maier N, et al. Human Cerebrospinal Fluid Monoclonal N-Methyl-D-Aspartate Receptor

\section{FUNDING}

This work was supported in part by grants from the National Natural Science Foundation of China (81802088), the Joint Funds for the Innovation of Science and Technology, Fujian Province (2018Y9080), and the Startup Fund for scientific research, Fujian Medical University (2019QH1094).

\section{ACKNOWLEDGMENTS}

We thank all patients for their data and blood donation and all teachers at the Department of Laboratory Medicine, the First Affiliated Hospital of Fujian Medical University for their help during the study.

Autoantibodies Are Sufficient for Encephalitis Pathogenesis. Brain (2016) 139:2641-52. doi: 10.1093/brain/aww208

16. Dubey D, Pittock SJ, Kelly CR, McKeon A, Lopez-Chiriboga AS, Lennon VA, et al. Autoimmune Encephalitis Epidemiology and a Comparison to Infectious Encephalitis. Ann Neurol (2018) 83:166-77. doi: 10.1002/ana.25131

17. Armangue T, Spatola M, Vlagea A, Mattozzi S, Cárceles-Cordon M, MartinezHeras E, et al. Frequency, Symptoms, Risk Factors, and Outcomes of Autoimmune Encephalitis After Herpes Simplex Encephalitis: A Prospective Observational Study and Retrospective Analysis. Lancet Neurol (2018) 17:760-72. doi: 10.1016/s1474-4422(18)30244-8

18. Titulaer MJ, McCracken L, Gabilondo I, Armangué T, Glaser C, Iizuka T, et al. Treatment and Prognostic Factors for Long-Term Outcome in Patients With Anti-NMDA Receptor Encephalitis: An Observational Cohort Study. Lancet Neurol (2013) 12:157-65. doi: 10.1016/s1474-4422(12)70310-1

19. Dubey D, Toledano M, McKeon A. Clinical Presentation of Autoimmune and Viral Encephalitides. Curr Opin Crit Care (2018) 24:80-90. doi: 10.1097/ MCC.0000000000000483

20. Ti D, Hao H, Tong C, Liu J, Dong L, Zheng J, et al. LPS-Preconditioned Mesenchymal Stromal Cells Modify Macrophage Polarization for Resolution of Chronic Inflammation via Exosome-Shuttled Let-7b. J Transl Med (2015) 13:308. doi: 10.1186/s12967-015-0642-6

21. Zhang J, Xu X, Zhao S, Gong Z, Liu P, Guan W, et al. The Expression and Significance of the Plasma Let-7 Family in Anti-N-Methyl-D-Aspartate Receptor Encephalitis. J Mol Neurosci (2015) 56:531-9. doi: 10.1007/s12031-015-0489-6

22. Guan H, Singh UP, Rao R, Mrelashvili D, Sen S, Hao H, et al. Inverse Correlation of Expression of microRNA-140-5p With Progression of Multiple Sclerosis and Differentiation of Encephalitogenic T Helper Type 1 Cells. Immunology (2016) 147:488-98. doi: 10.1111/imm.12583

23. Zhu S, Zhang X, Guan H, Huang F, Wu L, Hou D, et al. miR-140-5p Regulates $\mathrm{T}$ Cell Differentiation and Attenuates Experimental Autoimmune Encephalomyelitis by Affecting CD4(+)T Cell Metabolism and DNA Methylation. Int Immunopharmacol (2019) 75:105778. doi: 10.1016/ j.intimp. 2019.105778

24. Wu X, Ye Y, Niu J, Li Y, Li X, You X, et al. Defective PTEN Regulation Contributes to B Cell Hyperresponsiveness in Systemic Lupus Erythematosus. Sci Trans Med (2014) 6:246ra99. doi: 10.1126/scitranslmed.3009131

25. Yin R, Jiang J, Deng H, Wang Z, Gu R, Wang F. miR-140-3p Aggregates Osteoporosis by Targeting PTEN and Activating PTEN/PI3K/AKT Signaling Pathway. Hum Cell (2020) 33:569-81. doi: 10.1007/s13577-020-00352-8

Conflict of Interest: The authors declare that the research was conducted in the absence of any commercial or financial relationships that could be construed as a potential conflict of interest.

Publisher's Note: All claims expressed in this article are solely those of the authors and do not necessarily represent those of their affiliated organizations, or those of the publisher, the editors and the reviewers. Any product that may be evaluated in 
this article, or claim that may be made by its manufacturer, is not guaranteed or endorsed by the publisher.

Copyright $\odot 2022$ Liu, Fan, Lin, Tang, Wang, Huang, Zhang, Chen and Ou. This is an open-access article distributed under the terms of the Creative Commons
Attribution License (CC BY). The use, distribution or reproduction in other forums is permitted, provided the original author $(s)$ and the copyright owner(s) are credited and that the original publication in this journal is cited, in accordance with accepted academic practice. No use, distribution or reproduction is permitted which does not comply with these terms. 